\title{
Image segmentation of acidity of agricultural lands in Eastern Siberia
}

\author{
Dmitriy Perfilyev ${ }^{1 *}$, Tatiana Avdyukova ${ }^{2}$ and Pavel Zakharov ${ }^{3}$ \\ ${ }^{1}$ Siberian Federal University, Institute of Space and Information Technologies, Russian Federation \\ 2Director (e-mail: agrohim_24_3@mail.ru)Station of Agrochemical Service 'Solyanskaya', \\ Pervomayskaya str., 19, vil. Novaya Solyanka, Russian Federation \\ ${ }^{3}$ Deputy Head of Library and Publications Department Siberian Federal University, Russian \\ Federation
}

\begin{abstract}
The paper proposes a mathematical model for segmentation of the acidity dynamics of agricultural lands in Eastern Siberia. The segmentation rule is based on the analysis of the image frequency characteristic acidity, which makes it possible to predict the change in the property of the segmented areas. The mathematical approach to the segmentation of the dynamics of agrophysical parameters of agricultural soils has proves to be effective in managing the quality of the natural energy state on the control plots. It is expected that the experience will be applied to the entire responsibility area of 'SAS' 'Solyanskaya' (912.4 thousand ha). Introduction and distribution of innovative practices and technologies will provide new opportunities for monitoring and management of the natural energy state quality of the East-Siberian territory (23 million ha).

Keywords: image segmentation, segmentation rule, segmentation of the dynamics of agrochemical parameters.
\end{abstract}

\section{Introduction}

The national food security problem cannot be solved without a profound digital transformation of technologies for monitoring and predicting the dynamics of key agrochemical parameters of soil productivity. New technologies should be focused on the optimal use and preservation of the natural energy reserve of agricultural lands.

The transformation of technology for monitoring and managing the quality of natural energy reserve of lands requires the widespread use of highly automated (smart) productivity quality management technologies. This should be based on mathematical analysis and prediction of the dynamics of key agrophysical parameters of soils.

Technologically, such transformation can be seen from the mathematical segmentation of the image.

The mathematical formulation of the segmentation problem is discussed in [1-6].

It is shown that the solution to the segmentation problem of the function $f(a)$ is to divide the image into $S=\{s 1, \mathrm{~s} 2, \ldots, \mathrm{sk}\}$, non-empty connected areas for which the LP rule takes true values only if any pair of elements from any subset of $s_{i} \in S$ satisfies the goal of interpreter.

Under alternative conditions, the goal is limited to estimating the dynamics of the distribution density of the segmented image properties.

In general, segmentation can be considered as:

\footnotetext{
* Corresponding author: perfilyev7775@yandex.ru
} 
Seg: $f(a) \stackrel{L p}{\longrightarrow} S^{(\text {Sem. })}$,

where:

- $S^{(\text {Sem. })}-$ semantic relations.

- $s_{i}^{(\text {Sem. })}$ - the name of the $s_{i} \in S$ area;

- $L P\left(s_{i}{ }^{S e m}\right)$ - the «neighbourhood» model.

In [20], there are the main forms of syntactic neighbourhood relations that are typical for the image area. For the "Area" model of the image $\left|s_{i}\right|>1$ [20]:

$$
s_{10}=<P_{i},\left\{b_{i}\right\}^{1} \cup\left\{b_{1}-b_{3}, b_{5}\right\}^{2}>,
$$

where:

- $b_{i}{ }^{1}-$ u-nary attribute of the $s_{i}$ area that includes a subset of the elements of the $P_{i}$. area. For example, it is the sign of the convexity of the shape of the area;

- $b_{1}{ }^{2}-b_{3}{ }^{2}, b_{5}{ }^{2}$ - models of possible neighbourhood relations of boundary elements of the area. For example, it is the cardinality of the boundary elements of a segmented area with other areas including the «background» area;

A satisfactory segmentation result for $\mathrm{S}$ set that is given is an ideal case and is suitable for the "classical" syntax of deterministic mapping of object properties in the image:

- the problem representation allows for setting in advance the value of the iconic function $f(a)$ for the «background» area. The "background" area fills the entire image which contains the $\mathrm{S}$ areas representing the properties of the object. The "background" area is defined by the most contrasting value of the function relative to the segmented areas, usually close to the maximum (minimum) value;

- the segmented $s_{i}$ areas have differences in u-nary features (usually in the gradient: $\nabla f(a))$ and neighbourhood, they are also true for the "background" area. The areas have a boundary in the form of a closed external and possibly internal contour in accordance with the set of neighbourhood models of the boundary elements of the $b_{1}{ }^{2}-b_{3}{ }^{2}, b_{5}{ }^{2}$ area.

In deterministic conditions, the LP rule is a comprehensive estimation of the $\nabla f(a)$ "distance" (proximity) of the $p_{i} \in f(a)$ current image element and the neighbourhood (adjacency) of the $p_{j} \in s_{i}$ form:

$$
\left|p_{i}: \nabla f(a)-s_{i}\right| \rightarrow \text { min., and } p_{i} \cap p_{j}=1, \text { где } p_{j} \in s_{i} .
$$

Thus, every current element of the $p_{i} \in f(a)$ image refers only to one $s_{i} \in S$ area. Accordingly, segmentation is performed on the basis of the uniformity and connectivity of the $f(a)$ image elements.

Without the ideal conditions for displaying the properties of an object, there are regular physical violations of the shape of segmented areas due to distortions in the form of incomplete, false, thickened contours, "overlapping" areas and other related errors $[1,10,11]$.

One of the ways to deal with errors is to modernize the rule (1) of the semantic component, as a configurable "radius" that allows for improving the quality of segmentation. Such a rule has the following form:

$$
\left|p_{i}: \nabla f(a)-L P\left(s_{i}^{\text {Sem }}\right)\right|<r_{i}^{\text {Sem }} \text { и } p_{i} \cap p_{j}=1, \text { где } p_{j} \in S_{i},
$$


where $r_{i}^{\text {Sem }}$ - the radius of uniformity for $s_{i}^{\text {Sem }}$.

Thus, the assessment of the uniformity of a class can be changed in the process of segmentation [21, 22], by changing the $r_{i}^{\text {Sem }}$ regarding the analysis of the uniformity of adjacent classes. In addition, an adjustment of the power $\mathrm{S}$ can be made on the basis of the analysis of the uniformity of $s_{i}^{S e m}$ and the power of the "background" area.

The use of the adaptation principle (2) on the one hand improves the quality and on the other hand naturally limits the possibility of applying the $L P\left(s_{i}{ }^{S e m}\right)$ rule when using image analysis systems with different properties and in different physical display conditions. Moreover, $L P\left(s_{i}^{\mathrm{Sem}}\right)$ usually represents a priori knowledge based on iconic information, which limits the practical use of formalization [1-4] in solving the problem of interpretation.

The way out of this situation is to recognize the need to consider the image in a purely mathematical aspect, as continuous and differentiable function $f(z)$, problem-subject area and interpretation of the characteristics of the attributes of segmented image areas [22, 23].

The image of the problem-subject area is based on the given physical attributes of the process (object) under study:

Given $F\left\{z_{1}, z_{2}, \ldots, z_{n}\right\}$-the complex of attributes of an object represented by a set of images $f\left(z_{i}\right)$, as well as the rules $\operatorname{Lp}\left(S_{i}^{\text {Sem }}\right)$ that determine the uniformity radius $r_{i}^{\text {Sem }}$ of the $i$ th attribute value.

The segmentation task can be solved by separating the function $f\left(z_{i}\right)$ into $N$ areas (classes) for which $\operatorname{Lp}\left(S_{i}^{\text {Sem }}\right)$ takes true values that comply with the objectives.

In this case, the segmentation is considered a continuous process of analysing the dynamics of the image $f\left(z_{i}\right)$ :

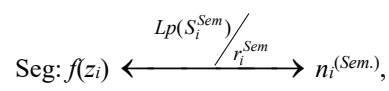

where $n_{i}^{(\text {Sem. })} \in N$ is the semantic relation (class name) defined in the analysis of the uniformity of the current state area $f\left(z_{i}\right)$, limited by the radius $r_{i}^{(\text {Sem. })}$.

The complexity of segmentation is determined by a number of conceptual aspects:

- interpretation objective -(object phenomenology);

- adequacy of imaging -(of phenomenological properties or essential attributes of the adopted model: $f(z))$.

In the general case, segmentation of the function $f(z)$ satisfies the evaluator when any pair of elements $\left[z_{i}-z_{i-1}\right]$ from any subset $z_{i} \in f(z)$ compiles the contour description.

$$
\oint_{C} f(z) d z=\lim _{\max \left[z_{i}-z_{i-1}\right] \rightarrow 0} \sum_{i=1}^{n} f^{\prime}\left(s_{i}\right)\left(z_{i}-z_{i-1}\right),
$$

where $f^{\prime}\left(s_{i}\right)$ describes a change in the physical attribute (semantic relationship, area name- $\left.S_{i}^{S e m}\right) ; L p\left[z_{i}-z_{i}-1\right]$ is the reference to the 'neighbourhood' model, defined by the $r_{i}^{(\text {Sem.) })}$ radius of the uniformity of the physical attribute.

The mathematical 'neighbourhood' model for any area of the function $f(z)$ of the closed loop is described by the Cauchy integral formula.

$$
f^{(i)}(z)=\frac{i !}{2 \pi i} \oint_{C S_{i}} \frac{f(z)}{\left(z_{0}-z\right)^{i+1}} d z
$$


If the path in the positive direction (counter clockwise) is defined by a smooth parametric representation $z=z(t)(\alpha \leq t \leq \beta)$, where $z(\alpha)=a$ and $z(\beta)=b(a, b$ are initial arc integration points), then the calculation is carried out according to the formula.

$$
f(z) d z=\oint_{\beta \alpha} f(z(t)) z^{\prime}(t) d t=\int_{\alpha}^{\beta} \operatorname{Re}\left[f(z(t)) z^{\prime}(t) d t+i \int_{\alpha}^{\beta} \operatorname{Im}\left[f(z(t)) z^{\prime}(t) d t\right.\right.
$$

Formula (3) allows for segmenting the contour of area $S_{i}$ on the image $f(z)$ and (4) describes the contour properties.

In case of a multiple connection (i.e. when bypassing the complement in the negative direction), the segmentation and description of the contour are slightly complicated.

$$
f^{(i)}(z)=\frac{i !}{2 \pi i} \oint_{C S_{i}} \frac{f\left(z_{0}\right)-f(z)}{\left(z_{0}-z\right)^{i+1}} d z
$$

Accordingly, the calculation is carried out by the formula:

$$
f(z) d z=\int_{\alpha}^{\beta} \operatorname{Re}\left[f(z(t)) z^{\prime}(t) d t+i \int_{\alpha}^{\beta} \operatorname{Im}\left[f(z(t)) z^{\prime}(t) d t+\sum_{s=1}^{n} \int_{s_{i}} \operatorname{Re}\left[f(z(t)) z^{\prime}(t) d t+i \int_{s_{i}} \operatorname{Im}\left[f(z(t)) z^{\prime}(t) d t\right.\right.\right.\right.
$$

It is known that the critical derivation point $z_{0}$ serves as the centre of the semantical radius $r_{i}^{(S e m .)}$. Usually, the value of the critical point is set empirically based on expert knowledge. For example, in the field of plant cultivation, the critical points represent the centres of four groups of cultivated plants with corresponding acidity values.

Conceptually, the value of the critical point is defined on the section of the segmented area boundary and the background where the integral equals to zero at any size and position of the common boundary.

For this reason, it is proposed to connect the position of the critical point with the dynamics of the image frequency characteristic. In this case, the bracketing $r_{i}^{(\text {Sem.) }}$ will ensure the uniformity of the frequency characteristic of the segmented image area.

\section{Method of analysis of the key soil productivity parameters}

The important parameters determining the energy state of agricultural lands in the eastern territories of Krasnoyarsk Territory are the following: acidity, moisture, content of humus, active forms of phosphorus and potassium on slightly acidic and neutral soils in the conditions of sharply continental climate of Siberia [16-19].

An example of the segmentation of images of weighted averages of acidity, active phosphorus and uncomplexed potassium for groups of plants on plots of agricultural land in the area of Stepnyaki village, Brazhensky village council, Kansk district, Krasnoyarsk Territory, is shown in Figure 1. 


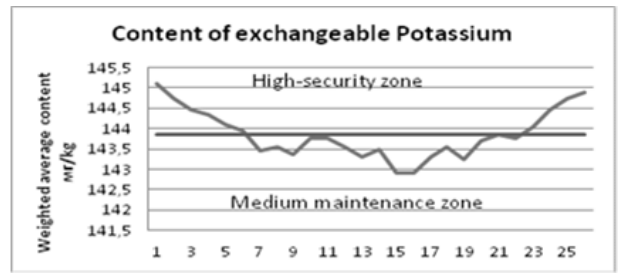

Weighted averages of the exchange potassium, experimental area

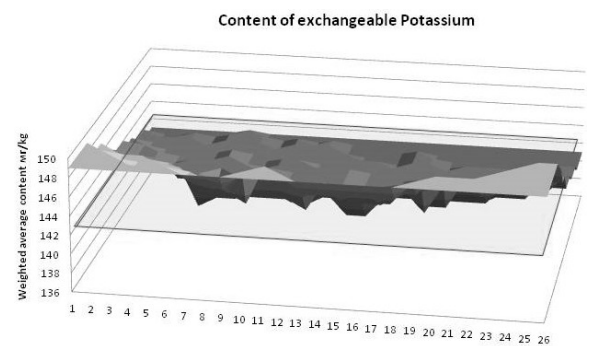

Image segmentation of the distribution of the weighted averages of the exchange potassium

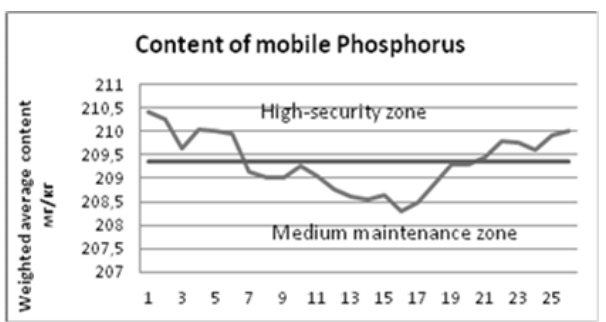

Weighted averages of the active phosphorus, experimental area

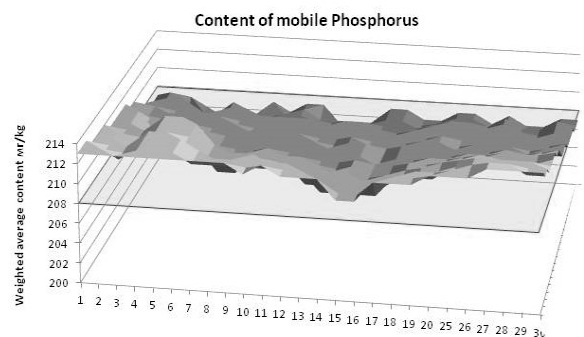

Image segmentation of the distribution of the weighted averages of the active phosphorus 


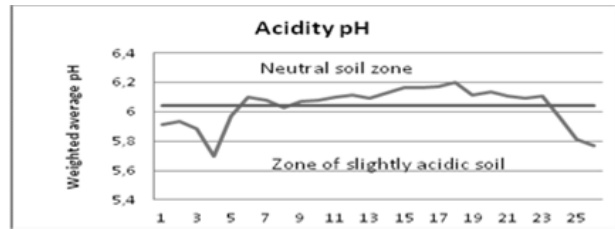

Weighted averages of acidity, experimental area

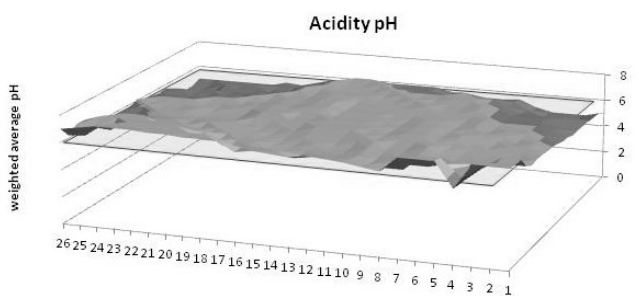

Fig. 1. Image segmentation of the distribution of the weighted averages of acidity

Usually, the interpretation of the object phenomenology is based on the analysis of the frequency, amplitude and phase characteristics of the segmented areas of the image $f\left(z_{i}\right)$.

The segmentation starts from the calculation of the centre line of the image graph $f\left(z_{i}\right)$ :

$$
\mu_{c p}=\frac{\sum_{i=1}^{k} \mu_{i}}{k}
$$

where $k$ is the number of samples.

The uniformity boundaries are calculated using the $L p$ rule as follows:

$$
L p_{U}=\mu_{c p}+\frac{r_{i}^{(\text {Sem })} \sigma}{\sqrt{n}}
$$

where $n$ is the number of measurements.

Deviation value $\sigma$ is calculated as follows:

$$
\sigma=\sqrt{\frac{\sum_{i=1}^{k} \sum_{j=1}^{n}\left[\mu_{i, j}-\mu_{c p}\right]^{2}}{n k}}
$$

The segmentation and contour description are carried out in a similar way in case of a multiple connection, i.e. with the integration over the upper and lower boundaries of the image area.

$$
L p_{U L}=\mu_{c p} \pm \frac{r_{i}^{(\text {Sem })} \sigma}{\sqrt{n}} .
$$


Apparently, the segmented areas (see Figure 1) by the $L_{p U L}$ rule should be considered relatively uniform since the segmentation is performed on the basis of a rather abstract parameter like a weighted average.

It is convenient to improve the quality of area segmentation and thus obtain a more accurate solution to the task based on the analysis of their frequency characteristic [20-23]. In this case, the segmentation based on the analysis of the frequency dynamics of the segmented image area $f\left(\omega_{r}\right)$ is described by an integral formula of the type [22,23].

$$
f^{(i)}\left(\omega_{0}\right)=\frac{i !}{2 \pi i} \oint_{S_{i}} \frac{f\left(\omega_{r}\right)}{\left(\omega_{r}-\omega_{0}\right)^{i+1}} d \omega_{r} .
$$

where: $\omega_{r}$ is the frequency assessment on the interval

$\left[\omega_{i}-\omega_{i-1}\right]$ typical of a slightly acidic medium with a radius of $r_{i}^{S e m} ; \omega_{0}$ - critical derivation point.

The integral characterising the change in the frequency of the segmented area is calculated using the formula.

$$
f\left(\omega_{r}\right) d \omega=\oint_{\beta \alpha} f\left(\omega_{r}(t)\right) \omega_{r}{ }^{\prime}(t) d t=\int_{\alpha}^{\beta} \operatorname{Re}\left[f\left(\omega_{r}(t)\right) \omega_{r}{ }^{\prime}(t) d t+i \int_{\alpha}^{\beta} \operatorname{Im}\left[f\left(\omega_{r}(t)\right) \omega_{r}{ }^{\prime}(t) d t\right.\right.
$$

Formula (4a) allows for segmenting the image area with a given frequency characteristic. The analysis of the frequency dynamics in conjunction with the amplitude analysis of dynamics, phase and image area parameters makes it possible to predict changes in the qualitative uniformity of physical parameters under known seasonality conditions and taking into account the quality of the input data.

Presumably, areas with a relatively high frequency index tend to transfer to the more active soil class and vice versa.

\section{Result analysis and discussion}

The data on the distribution of the agrophysical parameters on the basis of long-term research conducted by the staff of FSUE SAS 'Solyanskaya' provide a general image of the dynamics of agricultural land productivity in Kansk district of Krasnoyarsk Territory, in particular agricultural lands of Stepnyaki village, Brazhensky village council, Kansk district, Krasnoyarsk Territory.

The calculation of the uniformity radius $r_{i}^{S e m}$ for the $i$-th class is carried out from the critical point $\omega_{0}$ as follows:

$$
\omega_{0 i}=\mu_{c p} \pm \frac{r_{i}^{\left({ }^{(S e m}\right)} \sigma}{\sqrt{n}} .
$$

It should be noted that the value of the 'radius' $r_{i}^{\text {Sem }}$ is described by a graph $f\left(\omega_{r}\right)$. As a result, the characteristic $f\left(\omega_{r}\right)$ makes it possible to form the classes of image areas with the radius $r_{i}^{\text {Sem }}$, which reflect the uniform object properties (3a).

The calculation of the contour area of the segmented areas is carried out according to 
(4a).

Calculation of the contour frequency:

$$
f\left(\omega_{r}\right)=P(\omega)+j Q(\omega)=f(\omega) e^{j \varphi(\omega)}=f(\omega)[\cos \varphi(\omega)+j \sin \varphi(\omega)],
$$

where: $P(\omega)=\operatorname{Re} f(j \omega)$;

$$
\begin{aligned}
& Q(\omega)=\operatorname{Im} f(j \omega)=f(\omega) \sin \varphi(\omega)] ; \\
& \varphi(\omega)=\operatorname{arctg} \frac{P(\omega)}{Q(\omega)} .
\end{aligned}
$$

Figure 2, shows the result of the image area segmentation that makes it possible to analyse the dynamics of three acidity classes of soils (slightly acidic, circumneutral and neutral) of the Stepnyaki village agricultural land plot.

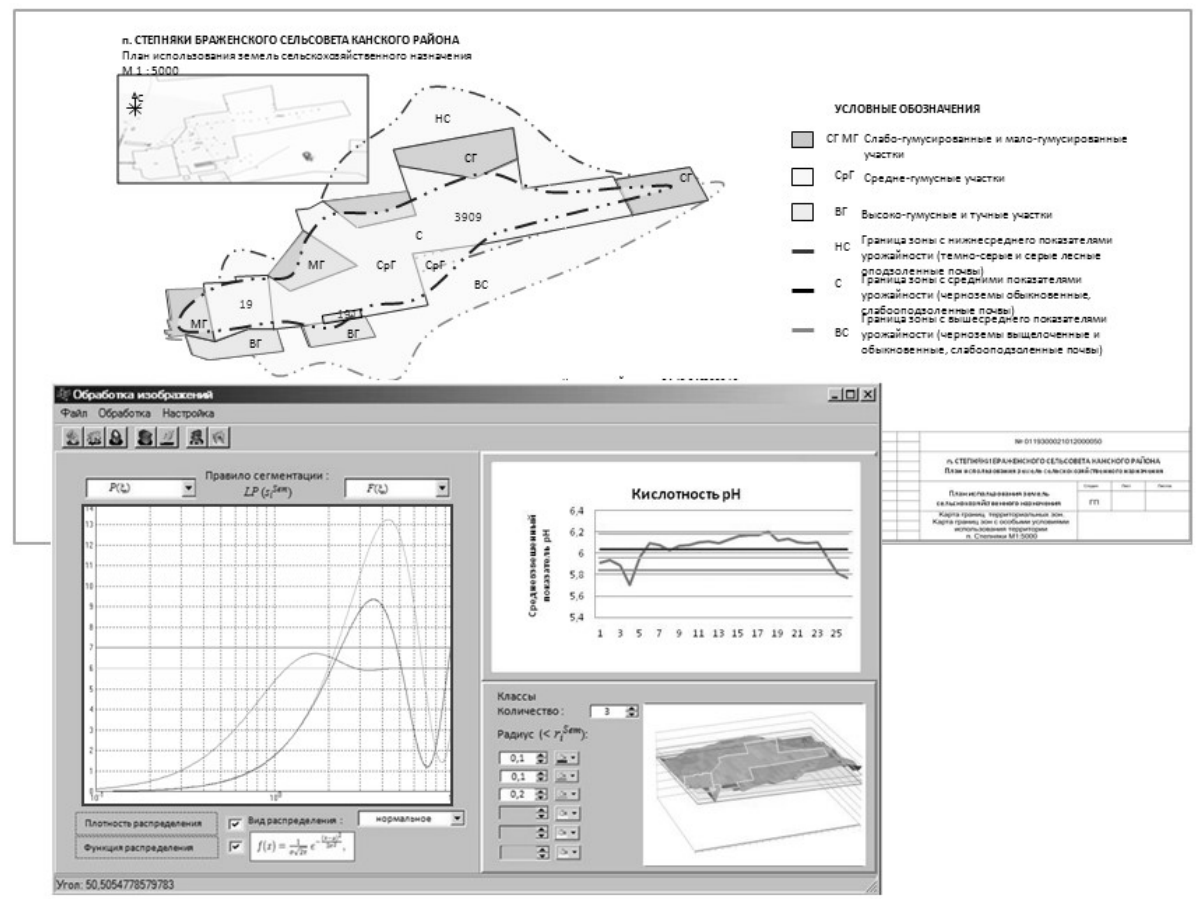

Fig. 2. Segmentation the image frequency properties and evaluation of the uniformity of segmented area classes

The central segmented area covers the entire study area of agricultural land in Stepnyaki village. This area belongs to the 'circumneutral' soil class ( $\mathrm{pH}$ 5.9), which is relatively to the north of more acidic soils and to the south of neutral soils.

According to further analysis of the central plot in relation to the density of image frequency characteristic $f\left(\omega_{r}\right)$, the northern part of the plot, with its relatively high 
frequency parameter, tends to lower the acidity of the medium to a slightly acidic soil ( $\mathrm{pH}$ 5.6), while the southern part accordingly tends to increase the acidity to a neutral medium ( $\mathrm{pH}$ 6.2) (as shown in Figure 2. on the right). This is also confirmed by the evaluation of the density of the image frequency characteristic $f\left(\omega_{r}\right)$ of the central plot (Figure 2. on the left).

Overall, the prognosis based on the analysis of the image frequency dynamics shows a relatively high qualitative uniformity of the central plot; the robustness of the plot in maintaining a 'circumneutral' environmental response serves as a good complement to standardised methods of analysis of soil condition of agricultural lands.

\section{Conclusion}

The proposed mathematical model is harmonised with the accepted methods used to analyse the main agrochemical parameters [24-28] and greatly enhances the possibilities for more accurate analysis, interpretation and prediction of agrophysical characteristics of soil condition.

The segmentation mathematical model based on more complex 'neighbourhood' relations defined by the uniformity radius $r_{i}^{\text {Sem }}$ of the image area frequency characteristic $f\left(\omega_{r}\right)$ allows for performing the fragmentation of the characteristic into $N$ classes under causal conditions, for which $\operatorname{Lp}\left(S_{i}^{S e m}\right)$ takes true values that comply with the objectives.

In this case, object segmentation is a combined strategy:

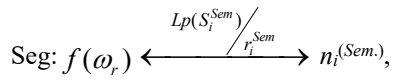

where $r_{i}^{(\text {Sem.) }} \in N$ is the semantic relation (object name) determined during the analysis of the frequency uniformity of the segmented area boundaries.

The mathematical approach to the segmentation of the dynamics of agrophysical parameters of agricultural soils has proved to be effective in managing the quality of the natural energy state on the control plots. It is expected that the experience will be applied to the entire responsibility area of 'SAS' 'Solyanskaya' (912.4 thousand ha).

In the future, it is expected to create a 'Digital atlas' reflecting the dynamics of changes in the main agrochemical parameters of soils as part of the Project 'Strategy for the Development of the Agroindustrial Complex of Krasnoyarsk Territory ( 5 million ha) for the period until 2030 (http://www.krskstate.ru/2030/plan).

Introduction and distribution of innovative practices and technologies will provide new opportunities for monitoring and management of the natural energy state quality of the East-Siberian territory (23 million ha).

\section{References}

1. D. A. Denisov Computer aided video analysis methods: monograph. - Krasnoyarsk State Technical University Krasnoyarsk, 1993. - 192 p. (1993)

2. P. A. Chochia Pyramidal algorithm of image segmentation // Information processes. Pub. by Moscow State Technical University named after N. E. Bauman. 2010. vol. 10, No. 1.-P. 23-35. (2010) 
3. S. Minaee, S., Y. Y. Boykov, F. Porikli, A. J. Plaza, , Kehtarnavaz, N., \& Terzopoulos, D. Image segmentation using deep learning: A survey. IEEE Transactions on Pattern Analysis and Machine Intelligence. (2021).

4. M. Brand, V. Kettnaker Discovery and segmentation of activities in video //IEEE Transactions on Pattern Analysis and Machine Intelligence. - 2000. - T. 22. - №. 8. - C. 844-851. (2000).

5. S.V. Belim, S.B. Larionov. Segmentation of images on the basis of algorithm of communities separation on the graph // Mathematical structures and modeling 2016. № 3(39). P. 74-85 (2016).

6. G. Khanykov Classification of image segmentation algorithms // The news universities. Instrumentation. 2018. T. 61, № 11. P. 978-987. (2018).

7. L. S. Avdyushkina, Two-stage method nature texture classification // Management processes and sustainability 2016 3(1): P. 332-336 (2016).

8. D.N. Gonçalves, V.A. de Moares Weber, J.G. Bragato Pistori, R.d. Costa Gomes, A.V. de Araujo, M.F. Pereira, W.N. Gonçalves, H. Pistori, Carcass image segmentation using CNN-based methods, Information Processing in Agriculture (2020), doi: https://doi.org/10.1016/j.inpa.2020.11.004

9. R. Srikanth and K. Bikshalu, Multilevel thresholding image segmentation based on energy curve with harmony Search Algorithm, Ain Shams Engineering Journal, https://doi.org/10.1016/j.asej.2020.09.003

10. Essam H. Houssein, Bahaa El-dinHelmy, Diego Oliva, Ahmed A.Elngar, Hassan Shaban «A novel Black Widow Optimization algorithm for multilevel thresholding image segmentation» Expert Systems with Applications Available online 4 November 2020, 114159

11. Prakash Purswani, Zuleima T. Karpyn, Khaled Enab, YuanXue, XiaoleiHuang «Evaluation of image segmentation techniques for image-based rock property estimation» Journal of Petroleum Science and Engineering Volume 195, December 2020, 107890

12. TaoWang. ZexuanJi JianYang QuansenSun XiaoboShen ZhenwenRen QiGe «Label Group Diffusion for Image and Image Pair Segmentation» Pattern Recognition Available online 17 December 2020, 107789, 107789

13. Brian L. DeCost, Elizabeth A. Holm A computer vision approach for automated analysis and classification of microstructural image data // Computational Materials Science 110. 2015. P. 126-133. (2015).

14. S. Khodaskar, S. Ladhake Semantic Image Analysis for Intelligent Image Retrieval International Conference on Intelligent // Computing, Communication \& Convergence (ICCC-2014) Institute of Management and Technology, India 2014, P. 193-197. (2014).

15. S. Banerjee, S. K. Ghosh, S. Datta, S. K. Saha Segmentation of dual phase steel micrograph: An automated approach // Measurement 2013. P. 2435-2440. (2013).

16. T.V. Avdyukova, S.A. Hrutsky. Fertility State of Arable Lands in the East Zone of Krasnoyarsk Krai // Agriculture. 2017. № 1. C. 25-29. (2017).

17. T. A Milovskih., T. A Vyatkina., T. N. Smirnova Productivity of agricultural land in Sakhalin Oblast conditions // Scientific and technical achievements of AIC, 2019. Vol. 33. No. 4. P. 53-56. DOI: 10.24411/0235-2451-2019-10413. (2019).

18. V.V. Chuprova, A.A. Belousov, E.N. Belousova, Yu.V. Gorbunova Evaluation of agroecological condition of the soils involved in the develop-ment of sand and gravel quarries of Kansk district of Krasnoyarsk region // Journal of Siberian Federal University. Agronomic 2019. № 3, 16-21. (2019).

19. Yu. P. Tandelov Productivity of acidic soils in the agricultural area of Krasnoyarsk Territory / Yu. P. Tandelov. - Krasnoyarsk, 2012. - 161 p. (2012). 
20. Dmitry A. Perfil'ev Segmentation Object Strategy on Digital Image // Journal of Siberian Federal University. Engineering \& Technologies, 2018, 11(2), 213-220. (2018).

21. D.A. Perfilyev Object segmentation on a digital image // Regional issues of earth remote sensing; materials of international scient. conf./scient. Rev. by E. A. Vaganov; resp. for rel. A.V. Mashukova - Krasnoyarsk; Sib. Fed. Univ., 2018, 366 p. ISBN 978-5-76383109-2. (2018).

22. D.A. Perfilyev Strategy of object segmentation on a digital image // Regional issues of earth remote sensing; materials of VI intern. scient. conf., Krasnoyarsk, September 10 - 13, 2019/scient. rev. by E. A. Vaganov; resp. edit. G. M. Tsibulsky. - Krasnoyarsk; Sib. Fed. Univ., 2019, 368 p. ISBN 978-5-7638-4176-3. (2019).

23. D.A. Perfilyev Segmentation of frequency nonuniformity of the image // Regional issues of earth remote sensing; materials of VII intern. scient. conf. Krasnoyarsk, September 29 - 2 October 2020/scient. rev. by E. A. Vaganov; resp. edit. G. M. Tsibulsky. Krasnoyarsk; Sib. Fed. Univ., 2020 - 328 p. ISBN 978-5-7638-4389-7. (2020).

24. GOST 26269-91, Soils. General requirements for the fulfilment of analyses Pub.by M.: Standard publishing, (1992).

25. GOST 26204-91, Soils. Determination of mobile compounds of phosphorus and potassium by Chiricov method modified by CINAO. Pub.by M.: Standard publishing, (1992).

26. GOST 26205-91, Soils. Determination of mobile compounds of phosphorus and potassium by Machigin method modified by CINAO M.: Standard publishing, (1992).

27. GOST 26207-91, Soils. Determination of mobile compounds of phosphorus and potassium by Kirsanov method modified by CINAO M.: Standard publishing, (1992).

28. GOST 26483-85, Soils. Preparation of salt extract and determination of its $\mathrm{pH}$ by CINAO method M.: Standard publishing, (1985). 